\title{
THE SLOPE OF THE PSYCHOMETRIC FUNCTION AT DIFFERENT WAVELENGTHS
}

\author{
LAURENCE T. MALONEY* \\ Department of Psychology, University of Michigan, 330 Packard Road, Ann Arbor, MI 48104, U.S.A.
}

(Received 10 June 1987; in revised form 11 May 1989)

\begin{abstract}
Many current models of visual detection predict that the slope of psychometric functions for detection will be independent of the spectral power distribution of the test light once the spatial and temporal characteristics of the light are fixed. This article examines reports that the slope of the psychometric function depends on the wavelength of a spectrally-narrowband test light of fixed size and duration. Three sources of difficulty that any experimental measurement of slope must encounter are addressed: (1) the different spatial distributions of the photoreceptor classes across the retina; (2) possible variations in threshold over the course of the experiment; and (3) the large variability of estimates of slope and the need for a method of assessing this variability. Measurements of slope with 2 and 4 deg test flashes against a bright $510 \mathrm{~nm}$ field show no significant trend with the wavelength of the test. A novel statistical test bounds the magnitude of possible variations in slope across the visible spectrum.
\end{abstract}

Color vision Psychometric function

\section{INTRODUCTION}

An important goal of color science is to devise a set of rules that predict the visibility of a test light from knowledge of its spectral power distribution. The common approach to this problem consists of two parts. First, the visual response is characterized in terms of the responses of a set of visual channels. Each channel is assumed to be a filter whose spectral sensitivity is a linear recombination of estimated cone photoreceptor sensitivities. Second, a rule is specified for combining the responses from separate visual channels into a single value estimating the detectability of the stimulus for the observer. When the channel spectral sensitivities and the rule of combination are specified, the model predicts the observer's sensitivity to lights with arbitrary spectral power distributions. A complete analysis requires in addition that we include the nonlinearities introduced by the observer's state of adaptation (see Stiles, 1978). The usual assumption is that the state of adaptation governs the weightings of the linear recombination of receptor signals contributing to the channels but that once a state of adaptation is fixed the channels themselves behave as linear systems.

*Reprint requests should be addressed to: Laurence T. Maloney, Department of Psychology, New York University, 6 Washington Place, 8th Floor, New York, NY 10003, U.S.A.
Stiles (1939, 1978), for example, developed an empirical procedure for specifying the spectral sensitivities of the underlying visual channels using the method of selective light adaptation. In later work, Boynton, Ikeda and Stiles (1964) found that the spectral sensitivities inferred from the light adaptation experiment could not be used to explain test visibility without postulating considerable interactions among the channels in the rule of combination.

The most-commonly considered rules of combination take the following form for $N$ channels:

$$
r=\sum_{k=1}^{N} c_{k}(L)^{\rho_{k}}
$$

where $r$ is the overall response, $c_{k}(L)$ is the $k$ th channel's response to the light $L$, and the exponents $\rho_{k}$ may differ. Stiles $(1939,1978)$, for example, postulated 5-7 independent channels. With proper choice of exponents $\rho_{k}$, equation (1) can also approximate probability summation among channels (Graham \& Robson, 1987). When the exponents $\rho_{k}$ are equal, then $r^{1 / \rho}$ is a norm in the three-dimensional space whose coordinate axes are the response values of the three channels. When all of the exponents $\rho_{k}$ are set equal to two, the norm is Euclidean and equally detectable stimuli lie on spheres. Models for which the rule of combination is a norm will be referred to as geometric models.

Guth (1965) and his colleagues (Guth, Donley \& Marrocco, 1969; Guth \& Lodge, 1973; Guth, Massof \& Benzschawel, 1980) developed a geo- 
metric model of the visibility of test lights in which the spectral sensitivity of the channels is estimated directly from test sensitivities. In this formulation as well as in the similar formulation by Ingling (Ingling, 1977; Ingling \& Tsou, 1977) the rule of combination is the Euclidean norm. Kranda and King-Smith (1979) develop an approach in which, again, the spectral sensitivities of the postulated channels are derived from the test mixture data themselves. Their approach differs from that of Guth and his colleagues in two ways. First, the number of channels is not limited to three, but rather guided by the fit to the data (they suggest that four channels are required). Second, the exponents for the rule of combination are assumed equal, but estimated to be approximately four. Consequently, the norm posited is non-Euclidean.

The choice of exponents in equation 1 directly affects observers' measured performances. The observers' performance is summarized as a psychometric function: the probability of detection as a function of $\log$ intensity, $I$. The individual data points on the psychometric function are usually analytically approximated from detection data by fitting a two-parameter function, such as a cumulative Gaussian or Weibull distribution (Weibull, 1951; Quick, 1974), to the data. The Weibull, whose equation is $P_{D}=1-\mathrm{e}^{-(l /)^{b}}$ is used in this article. $P_{D}$ is the probability of correct detection, $\alpha$ is a location parameter, and $\beta$ is a scale parameter that determines the slope of the function in semi-log coordinates. To represent the joint effect of the many experimental parameters, including the spatial, spectral, and temporal characteristics of the stimulus as well as the observer's adaptational state, by only two parameters, $\alpha$ and $\beta$, enormously simplifies the representation.

Geometric models share the prediction that slope parameier $\beta$ will be independent of the spectral power distribution of the test light (Massof, 1981). If the exponents are different for different channels, on the other hand, $\beta$ will change with the identities of the channels mediating detection. Consequently, if variations in the spectral power distribution of a test light result in variations in the measured slope of the psychometric function for detection, we may reject the class of geometric models.

If the spatial and temporal characteristics of stimuli are permitted to vary, then experimental results indicate that the obtained slopes of psychometric curves can vary as well. Cowan, Wyszecki and Yaguchi (1984), for example, report forced-choice measurements taken under conditions intended to isolate the luminance and the chromatic opponent mechanism in turn by proper choice of the timecourse and spatial frequency composition of stimuli. Their stimuli were spectrally broadband. With a "spatial and temporal profile for the test stimulus that rendered the achromatic channel insensitive". They report a slope value of about 2.5 , and with a spatial and temporal profile which rendered the chromatic channel selectively insensitive, they report a slope value of about 3.5. Ingling, Martinez and Lewis (1983) describe changes in the slope of the psychometric function with changing flash duration. Wandel', (Wandell, 1985; Wandell, Ahumada \& Welsh, 1984) reports typical slope values of about 2 for $1.85 \mathrm{deg}$ spectrally narrowband test stimuli with Gaussian timecourse while Nachmias (1981) reports that slope values are typically about 3 in experiments involving forced-choice detection of sine wave gratings. Wandell notes that his values are significantly lower than those gotten by Nachmias and others with spectrally broadband sine wave gratings.

The results above leave open the possibility that it is solely variation in spatial and temporal parameters that results in changes in slope. That is, once the spatial and temporal characteristics of the stimulus are fixed, the channels mediating detecting in equation (1) share a common fixed value of $\rho$ that is unaffected by the spectral power distribution of the stimulus. This hypothesis will be referred to as the spectral homogeneity hypothesis. The spectral homogeneity hypothesis is a statement about the relationship between the processing of information in chromatic vision and in spatiotemporal vision. If it holds, then it plausible to formulate geometric models of chromatic vision for stimuli of fixed spatiotemporal characteristics, and then determine how variation in the spatiotemporal characteristics alter the geometric model obtained. If it does not hold, geometric models lose much of their interest as theoretical tools (Massof, 1981).

What evidence is there, that the slope of the psychometric function varies when the spatiotemporal characteristics of the stimulus are fixed? Several authors have attempted to measure the slope of the psychometric function for spectrally narrowband lights of fixed spatiotemporal characteristics, varying only in wavelength across the visible spectrum. However, apart from work by Blackwell (1963) and Massof (1981), evidence for or against the spectral 
homogeneity hypothesis is primarily based on frequency-of-seeing responses. Crozier (1950) found a periodic dependence of slope on wavelength at foveal absolute threshold. His highest recorded slopes occurred at $434.5 \mathrm{~nm}$ and $712 \mathrm{~nm}$. Dillon and Zegers (1958) also measured foveal absolute threshold. Four out of five observers, measured at 31 wavelengths, exhibited statistically significant linear trends with lower slopes at shorter wavelengths, the fifth showed no trend. The magnitude of the trend is small as can be seen in Massof's replot of their data (Massof, 1981, Fig. 5). Bouman and Walraven (1957) collected frequency-of-seeing data in the course of their color naming experiment and remark that, for wavelengths near $550 \mathrm{~nm}$, the slopes of the functions are slightly less steep than for those in the red. Kranda and KingSmith (1979) note that the slope of the frequency-of-seeing functions they obtained with a white background and a $440 \mathrm{~nm}$ test was significantly lower than those at 550,580 and $680 \mathrm{~nm}$. They report no other significant differences.

But, as Nachmias (1981) has shown (and Bouman ard Walraven note), the frequency-ofseeing function does not provide an acceptable procedure from which to judge the stability of the slope of the psychometric function. Frequency-of-seeing curves have higher slopes and thresholds than force-choice psychometric functions and are affected by changes in observers' criteria, that is, shifts in criterion can mimic changes in slope. There are two previous studies that held spatiotemporal factors constant and measure the psychometric function across the visible spectrum by forced-choice methods, Blackwell (1963) and Massof (1981).

Blackwell (1963) presents measurements of the slope of the psychometric function at absolute threshold, for a $1 \mathrm{~min}$ arc $100 \mathrm{msec}$ test light, using a forced-choice method. His data (his Table 3) show no obvious trend with wavelength. Massof (1981) presents evidence indicating that the slope of the psychometric function at absolute threshold changes with changing wavelength. He measured probability of detection at absolute threshold as a function of wavelengths using a two-alternative, forcedchoice method. The test light was circular, $20 \mathrm{~min}$ in dia., of $50 \mathrm{msec}$ duration. Massof concluded that "... the shallowest slopes occurred in the short wavelengths, the steepest slopes occurred in the middle portion of the spectrum, and the slopes were of ... intermediate value in the long wavelengths." Note that his results are inconsistent with Crozier's frequency-of-seeing results, and Bouman and Walraven's results described above. They agree with Kranda and King-Smith's results on a white background for $440 \mathrm{~nm}$; they agree in direction but not in magnitude of trend with Dillon and Zegers results at short wavelengths.

There are problems with the analysis of the data in Massof's study. First, the conclusions are based on aggregate data across six observers, but an examination of the results for each observer alone (his Fig. 11) shows considerable individual variation. The analysis of variance performed confounds wavelength with observer: different observers took data at different wavelengths. Consequently, the significant difference in slope across wavelength reported could be simply a significant difference in observers' behavior. Further, the results for the two most experienced observers show the least trend across wavelengths. This last effect may be related to the use of a small test flash in foveal viewing as discussed next.

The short-wavelength cone retinal mosaic is relatively coarse in the central fovea and the central $25 \mathrm{~min}$ of the fovea is apparently devoid of functioning short-wavelength cones (Williams, MacLeod \& Hayhoe, 1981a,b; Castano \& Sperling, 1982; Williams \& Collier, 1983; Williams, 1985). Willmer and Wright (1945) examined color matching with a $20 \mathrm{~min}$ field and determined that the central fovea is essentially tritanopic. They comment on "... the difficulty of locating the small test field on the fovea and maintaining it in that position for more than a very short time ... any deviation from direct fixation immediately caused a breakdown in the matches obtained."

If subjects maintained perfect fixation during the course of Massof's threshold measurements, then the above results suggest that detection with a 20 min test flash did not involve shortwavelength photoreceptors. It seems more likely, especially in light of Willmer and Wright's comment above, that subjects did not maintain perfect fixation. Note, in this connection, that Dillon and Zegers (1958) found that threshold estimates (method of limits) were more variable at short wavelengths for four out of five observers, consistent with the claim that small changes in fixation affect threshold more at short wavelengths than at long.

The consequence of "mixing" data from measurements corresponding to two psychometric functions with different threshold and a com- 
mon value of $\beta$ is to lower the estimated value of $\beta$. Monte Carlo simulations of an observer, whose performance is described by a mixture of two Weibull psychometric functions with common $\beta=2$ but different thresholds $\alpha$, illustrate this decrease in slope. The two thresholds $\alpha_{1}$ and $\alpha_{2}$ were chosen so that the average of $\log \left(\alpha_{1}\right)$ and $\log \left(\alpha_{2}\right)$ was always $9.0 \mathrm{log}$ quanta/ $/ \mathrm{deg}^{2} \mathrm{sec}$. That is, if $\log \left(\alpha_{1}\right)$ were 8.7 , then $\log \left(\alpha_{2}\right)$ was 9.3 , etc. The difference hetween the two values of $\log \left(\alpha_{1}\right)$ is called the lag. Values of lag ranged from 0.0 to 0.65 . For each value of lag, 20 measurements of the observer's psychometric function were simulated. For a single measurement, the simulated observer responded to 840 trials distributed evenly at the seven intensity levels 8.6 , $8.7, \ldots, 9.2$ chosen to bracket the average threshold. On half the trials, the simulated observer responded as if $\alpha_{1}$ were threshold, on half as if $\alpha_{2}$ were threshold. The number and distribution of trials is similar to the actual number and distribution of trials experienced by human observers in expt I below. The 20 values of slope $\beta$ estimated for each lag were averaged. These values are plotted vs lag in Fig. 1. Note that estimated $\beta$ drops to half its true value over the range of lags examined. Interestingly, estimates of log threshold deviated by less than $1 \%$ from 9.0, the average of the two true $\log$ thresholds. A difference of $0.5 \mathrm{log}$ units or more in sensitivity across the fovea is consistent with the conclusions of Williams et al. (1981b). Small changes in fixation then serve to mix psychometric functions with different thresholds.

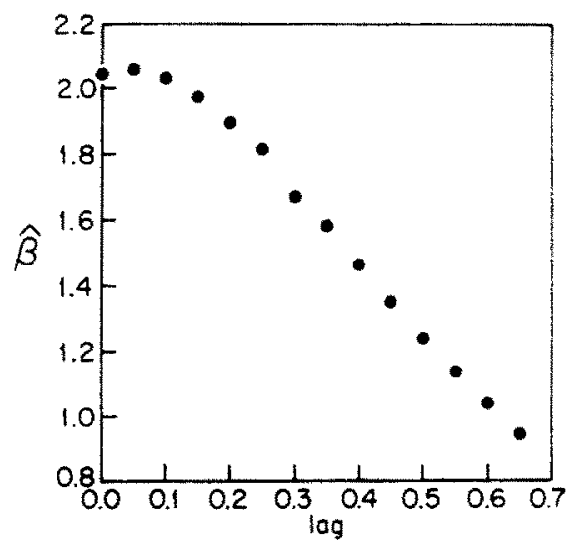

Fig. 1. The effect of mixing two psychometric functions with common threshold $\beta=2$ and values of $\log$ threshold that differ by $\log$ on the estimated value of $\beta$ returned by a maximum likelihood fit to the Weibull. Lag is plotted on the horizontal axis in $\log$ quanta/deg ${ }^{2} \cdot \mathrm{sec}$. On the vertical axis is plotted the mean of 20 estimates of $\beta$ obtained from twenty simulated measurements for a given lag. See text for details of the measurement conditions.
Lower values of $\beta$ found with small short-wavelength test probes, then, may be due solely to the relative sparseness of short-wavelength photoreceptors in central fovea combined with small failures of proper fixation.

Let me summarize the issues: the results of past tests of the spectral homogeneity hypothesis are in conflict. Massof's experiments do not resolve the question, at least until the problems raised above are addressed. Measurements of slope at short wavelengths are complicated by inhomogeneity in the distribution of photoreceptors in the fovea discussed above as well as the possibility of rod intrusion with larger test stimuli. A replication of Massof's measurements would not lay these last two issues to rest. Consequently, in the experiments reported below, no attempt is made to directly replicate Massof's experiment. Instead, these experiments will serve as independent tests of the spectral homogeneity hypothesis in the light adapted eye, using relatively large test flashes to minimize the effect of retinal inhomogeneity, and a bright $510 \mathrm{~nm}$ field to minimize rod involvement. The experiments will analyze individual Observers results, and will make use of a recently developed computationally-intensive method, the parametric bootstrap, for assessing the bias and standard deviation of parameters estimated by maximum likelihood techniques (Efron, 1979, 1982). The validity and usefulness of this method is assessed in Maloney (1989).

\section{METHODS}

\section{Stimuli}

The two series of measurements made are termed expts I and II. Both experiments were performed on a three-channel Maxwellian view system. Two channels were used for test lights of $50 \mathrm{msec}$ duration and either $2 \mathrm{deg}$ (expt I) or $4 \mathrm{deg}$ (expt II) in visual angle. The third channel was used to display a $510 \mathrm{~nm} 10 \mathrm{deg}$ adapting field of intensity $10.54 \log$ quanta/deg.sec (expt I) or $10.28 \mathrm{log}$ quanta/deg.sec (expt II). The three-channel Maxwellian view system used, and the procedures used to calibrate it, and to align Observers, is described in Wandell, Sanchez and Maloney (1982).

\section{Observers}

Two observers, KR with normal vision and LM with vision corrected to normal participated in the first experiment. KR was a paid 
research assistant. These two observers and two additional paid research assistants, EF with vision corrected to normal and $\mathrm{AO}$ with normal vision participated in the second experiment.

\section{Experimental procedure}

On the basis of Massof's results, the lowest slopes are to be found toward the blue end of the spectrum (near $440 \mathrm{~nm}$ ), the highest near $560 \mathrm{~nm}$, and intermediate values of slope at the long-wavelength end of the spectrum. The first experiment examined the slope of the psychometric function at four test wavelengths: 440 , 500,560 and $670 \mathrm{~nm}$.

In the first experiment, collection of data at different wavelengths was interleaved. Observer's completed 4-6 experimental sessions (with rest breaks) in the course of an hour on a single day. In each session, observers collected data for one of the test wavelengths. Before each session the observer adapted for three minutes to the $510 \mathrm{~nm} 10 \mathrm{deg}$ field. During the session, the observer was presented with 10 two-alternative, forced-choice trials for each of six intensities (spaced about 0.1 log units apart), a total of 60 trials. The choice of test-light and intensity on each trial were chosen at random by the computer controlling the apparatus. The range of intensities (different for each test light) were chosen to bracket the observer's threshold to that light, determined beforehand by a forcedchoice procedure staircase procedure. The observer requested each trial by pressing a button and proceeded through the 60 trials at his or her own pace. A session lasted about $7 \mathrm{~min}$ including the $3 \mathrm{~min}$ adaptation period. Half the sessions on a single day were devoted to the $560 \mathrm{~nm}$ test. The wavelengths used in the remaining sessions on a single day were randomly chosen from the remaining three wavelengths. Consequently, as much data was collected for $560 \mathrm{~nm}$ as the other three wavelengths combined.

The second experiment involved only three test wavelengths: 440,560 and $670 \mathrm{~nm}$. The objective of the second experiment was to accumulate 30 trials at each of seven intensity levels for a given wavelength within a single hour-long period. Each session consisted of 10 trials at each of 7 intensity levels for one wavelength. Three sessions constituted the needed 210 trials. Observers usually completed 3 or 6 sessions with rest breaks within an hour on a single day. Each of the paid research assistants practiced the task for at least 10 sessions over a 2 week period before collecting the data reported below.

\section{Data analysis}

For each of the two observers at each wavelength in the first experiment, all data was pooled to give one estimated psychometric function at wavelengths 440,500 and $670 \mathrm{~nm}$ and three estimated psychometric functions at $560 \mathrm{~nm}$. For each set of pooled data, the maximum likelihood esimates of the parameters $\alpha$ and $\beta$ of the Weibull model was estimted using STEPIT (Chandler, 1965) and the procedure described by Watson (1979). Next, confidence intervals were computed (based on 400 parametric bootstrap replications) for each estimated psychometric function in the first experiment, giving an estimate of bias and standard deviation for the slope parameter $\beta$ of each function as described in Maloney (1989). The estimated bias was subtracted from the experimentally obtained values of the slope $\beta$ and an approximate $95 \%$ confidence interval for the estimate slope $\beta$ was taken to be \pm 2 SD. The error bars in the figures for expt I represent these $95 \%$ confidence intervals.

For each observer and each wavelength in the second experiment, all 210 trials were pooled and fit to give a single estimated threshold $\alpha$ and slope $\beta$. There were, thus, several estimates of $\alpha$ and $\beta$ for each observer and wavelength based on 210 trials each (two functions for LM were based on 300 and 350 trials respectively). Bootstrap estimates of bias and variability were based on the median value of obtained estimates for $\alpha$ and the median value of estimates of $\beta$ (one estimate of bias and one estimate of standard deviation for each observer and each wavelength). The distribution of $\hat{\beta}$ for 210 trials per psychometric function is very skewed and consequently the 2.5th and 97.5 th percentiles of the bootstrap distribution were used as a $95 \%$ confidence interval.

\section{RESULTS AND DISCUSSION}

\section{Experiment I (2 deg test flash)}

In expt I, each psychometric function contains $480-840$ trials taken over the course of 2-5 days. Estimates of $\beta$ for each of four wavelengths for each observer are shown in Fig. 2. The vertical bars delimit the $95 \%$ confidence intervals computed using the parametric bootstrap method. The estimated confidence intervals are quite large compared to the differences in slope across wavelengths. Data were obtained on three observers for three functions at $560 \mathrm{~nm}$ 

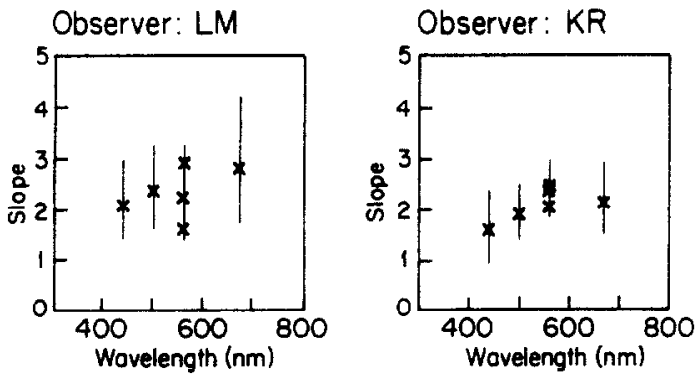

Fig. 2. Estimates of $\beta$ for each of four wavelengths for both observers. The vertical bars delimit the $95 \%$ confidence intervals ( $\pm 2 \mathrm{SD}$ ) computed using the bootstrap method. Where more than one estimate of $\beta$ is available for a single observer at a single wavelength, I base the confidence interval on the median values of these estimates.

and the spread of these three replications is also rather large and consistent with the estimated intervals. Slopes for $\mathrm{LM}$ at $560 \mathrm{~nm}$ range from 1.57 to 2.87 , for KR from 2.04 to 2.42 . There is no indication that the slope at $670 \mathrm{~nm}$ is lower than those at $560 \mathrm{~nm}$ for either subject. The slope values at $560 \mathrm{~nm}$ for each subject bracket the obtained values at $670 \mathrm{~nm}$; the confidence intervals overlap substantially. For subject LM, the values of slope at 440 and $500 \mathrm{~nm}$ are also bracketed within the values obtained at $560 \mathrm{~nm}$. There is no trend to lower slopes at shorter wavelengths. For subject $K R$, the value at $440 \mathrm{~nm}$ is below those for $560 \mathrm{~nm}$, but again, the estimated variability of the slope measurements do not permit us to reject the spectral homogeneity hypothesis. The slope at $440 \mathrm{~nm}$ is 1.56 , the median slope at $560 \mathrm{~nm}$ is 2.36 , approx. $34 \%$ lower. The data provide no support for a decrease in slope at longer wavelengths and little support for a decrease at shorter wavelengths given the variability of estimates of $\beta$. The magnitude of the decrease for KR $(34 \%)$ is considerably smaller than the decrease reported by Massof (1981) for these wavelengths (recall that Massof's stimulus and adapting conditions were different from those of the present experiment). Note also that there is qualitative agreement between the bootstrap estimate of the $95 \%$ confidence interval and the spread of the data in the one case for each observer (at $560 \mathrm{~nm}$ ) where measurements were replicated, though, of course, no firm conclusions about variability can be drawn from three points.

The slopes and thresholds in expt I were estimated from data taken over a period of 2-5 days using a 2 deg test flash to minimize the effect of the differing distributions of photoreceptor classes in and near the fovea. Changes in threshold to any of the test lights over the course of the 2-5 day period during which data was taken could affect the estimate of $\beta$. As discussed above, variability in $\alpha$ ("mixing") results in lowered estimates of $\beta$. Of greatest concern is the possibility that threshold to short wavelength test flashes is more variable than threshold at other wavelengths. The resulting mixtures would exhibit lower values of $\beta$ at short wavelengths. Since peak short-wavelength sensitivity is found in a ring around the center of the fovea of $1 \mathrm{deg}$ radius, the $2 \mathrm{deg}$ test field may still be small in comparison with inhomogeneities in short-wavelength photoreceptor distribution.

In expt II, then, the slopes of observers' psychometric functions were estimated using larger (4 deg) test lights and data for a single wavelength was collected over as short a time period as possible to preclude the possibility that day-to-day changes in true threshold were larger for short-wavelength than long-wavelength lights. Independent simulation indicates that although estimates of $\beta$ obtained under these conditions will be highly variable and biased, the median of such estimates is a stable though biased estimate of true $\beta$. Since the intent is to test for equality with changing wavelength, a common bias term added to estimates is of no concern here. The important point is that measurements taken in this manner are not affected by changes in threshold over a period of days.

\section{Experiment II (4 deg test flash)}

Figure 3 contains the estimated slopes from expt II for each of the three observers. The error bars are bootstrap $95 \%$ confidence intervais about the unbiased median value of slope for each wavelength and observer. Estimates of slope under these conditions are biased and very variable. But, again, there is no indication in the results that slope at $670 \mathrm{~nm}$ is lower than at $560 \mathrm{~nm}$ for any observer. Slopes at $440 \mathrm{~nm}$ may show a trend to be lower than those at $560 \mathrm{~nm}$ and $670 \mathrm{~nm}$, but the difference is small compared to the variability of the measurements, and not significant. The trend is most striking for the least experienced observer AO suggesting that what trend remains may be due to lack of experience in fixating the short-wavelength test. The results for individual subjects in Massof (1981) also suggest that it is the less experienced observers that exhibit lower values of $\hat{\beta}$ at short-wavelengths. 

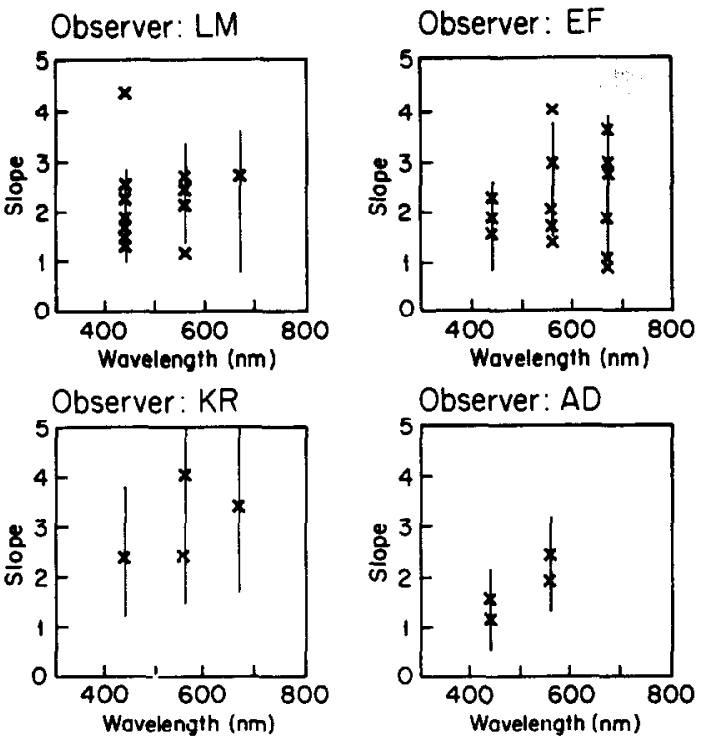

Fig. 3. Estimates of $\beta$ for each of four wavelengths for three observers. The vertical bars delimit the $95 \%$ confidence intervals computed using the bootstrap method on the median of the estimates for each observer, for each wavelength. The confidence intervals here are based on the 2.5 th and 97.5 th percentiles of the bootstrap replications since the actual distribution of $\hat{\beta}$ is markedly skewed with only 210 trials per psychometric function.

These results, of course, leave open the possibility that a different combination of adapting fields or choice of test stimulus could produce a significant difference in slope between the $440 \mathrm{~nm}$ test and the other test lights. It is important that this be demonstrated on single (not aggregate), experienced observers, with obvious confounds due to retinal inhomogeneity and rod intrusion excluded.

Since measurements of observers' slopes were repeated at selected wavelengths in both experiments, $i \imath$ is possible to assess the validity of the parametric bootstrap method by comparing observers' measured variability with the confidence intervals estimated by the bootstrap. The confidence intervals agree with the actual variability of the observers except for one point at $440 \mathrm{~nm}$ in expt II for observer LM. Estimates of slope of the psychometric function with the spacing and number of trials used in these experiments, are highly variable, and the size of the bootstrap confidence intervals are, if anything, understated (see Maloney, 1989, for a discussion).

\section{CONCLUSION}

Test stimuli with different spatial and temporal characteristics can produce psychometric functions with different slopes (Cowan et al., 1984). The differences are large, and apparently robust across experimental conditions: the sorts of stimuli used in spatial vision experiments produce slopes around 3-4, color vision stimuli produce slopes around 2-3 (Nachmias, 1981; Wandell, 1985).

It has proven to be more difficult to establish that test stimuli differing only in spectral power distribution can produce psychometric functions with different slopes. This spectral homogeneity hypothesis was tested in two experiments that used large test fields and a bright $510 \mathrm{~nm}$ adapting field for spectrally narrowband test lights. There were no statistically significant differences in slope across wavelength in either experiment; there appears to be a non-significant trend toward lower slopes at shorter wavelengths.

Acknowledgements-I wish to especially thank Brian Wandell who provided lab facilities, encouragement, and advice throughout. I also thank John Krauskopf, Denise Varner, and two anonymous reviewers for comments on an earlier draft, and Carl Ingling for comments and suggestions directed to an earlier presentation of this work.

\section{REFERENCES}

Blackwell, H. R. (1963). Neural theories of simple visual discriminations. Journal of the Optical Society of America, 53, 129-160.

Bouman, M. A. \& Walraven, P. L. (1957). Some color naming experiments for red and green monochromatic lights. Journal of the Optical Society of America, 47, 834-839.

Boynton, R. M., Ikeda, M. \& Stiles, W. S. (1964). Interactions among chromatic mechanisms as inferred from positive and negative increment thresholds. Vision Research, 4, 87-117.

Castano, J. A. \& Sperling, H. G. (1982). Sensitivity of the blue-sensitive cones across the central retina Vision Research, 22, 661-673.

Chandler, J. P. (1965). STEPIT. Indiana University, Bloomington, Indiana, U.S.A.: Quantum Chemistry Program Exchange, Department of Chemistry.

Cowan. W. B., Wyszecki, G. \& Yaguchi, H. (1984). Probability summation among color channels (Abstract). Optic News, 10, 90.

Crozier, W. J. (1950). On the visibility of radiation at the human fovea. Journal of General Physiology, 34, 87-136.

Dilion, D. J. \& Zegers, R. T. (1958). Quantal determination and statistical evaluation of absolute foveal luminosity thresholds and of threshold variability. Journal of the Optical Society of America, 48, 877-883.

Efron, B. (1979). Bootstrap methods: another look at the jackknife. The Annals of Statistics, 7, 1-26.

Efron, B. (1982). The jackknife, the bootstrap and other resampling plans. Philadelphia: Society for Industrial and Applied Mathematics. 
Graham, N. \& Robson, J. G. (1987). Summation of very close spatial frequencies: The importance of probability summation. Vision Research, 27, 1997-2007.

Guth, S. L. (1965). Luminance addition: General considerations and some results at foveal threshold. Journal of the Optical Society of America, 55, 718-722.

Guth, S. L. \& Lodge, H. R. (1973). Hetorochromatic additivity, foveal spectral sensitivity, and a new color model. Journal of the Optical Society of America, 63, $450-462$.

Guth, S. L., Donley, N. J. \& Marrocco, R. T. (1969). On luminance additivity and related topics. Vision Research, 9. 537-575.

Guth. S. L. Massof. R. W. \& Benzschawel. T. (1980). Vector model for normal and dichromatic color vision. Journal of the Optical Society of America, 70, 197-211

Ingling Jr, C. R. (1977). The spectral sensitivity of the opponent-colors channels. Vision Research. 17 , 1083-1090.

Ingling Jr, C. R. \& Martinez-Uriegas, E. (1985). The spatiotemporal properties of the $\mathrm{r}-\mathrm{g} \mathrm{X}$-cell channel.Vision Research, 25, 33-38.

Ingling Jr, C. R. \& Tsou, B. H.-P. (1977). Orthogonal combination of the three visual channels. Vision Reseurch. 17, 1075-1082.

Ingling $\mathrm{J}_{\mathrm{r}}, \mathrm{C}$. R. Martinez, E. \& Lewis, A. L. (1983). Tonic-phasic-channel dichotomy and Crozier's law. Journal of the Optical Society of America, 73, 183-189.

Kranda, K. \& King-Smith, P. E. (1979). Detection of coloured stimuli by independent linear systems. Vision Research, 19, 733-745.

Maloney, L. T. (1989). Confidence intervats for the parameters of psychometric functions. Perception and PSy. chophysics (in press).

Massof, R. W. (1981). Wavelength dependence of the shape of the foveal absolute threshold probability of detection functions. Vision Research, 21. 995-1004.
Nachmias, J. (1981). On the psychometric function for contrast detection. Vision Research, 21, 215-233.

Quick, R. F. (1974). A vector magnitude model of contrast detection. Kybernetik, 16, 65-67.

Stiles, W. S. (1939). The directional sensitivity of the retina and the spectral sensitivities of the rods and cones. Proceedings of the Royal Society, London, B127, 64-105.

Stiles, W. S. (1978). Mechanisms of colour vision. London: Academic Press.

Wandell, B. A. (1985). Color measurement and discrimination. Journal of the Optical Society of America, A 2 . $62-7 !$

Wandell, B. A., Sanchez, J. \& Maloney, L. T. (1982). Adaptation in the long-wavelength pathways. Vision Re search, 22, 1071-1074.

Wandell, B. A., Ahumada, P. \& Welsh, D. K. (1984). Reaction times to weak test lights. Vision Research. 24, 633640 .

Watson, A. B. (1979). Probability summation over time Vision Research, 19, 515-522.

Weibull, W. (1951). A statistical distribution function of wide applicability. Journal of Applied Mechanics. 18, 292-297.

Williams, D. R. (1985). Aliasing in human foveal vision. Vision Research, 25, 195-205.

Williams, D. R. \& Collier, R. (1983). Consequences of spatial sampling by a human photoreceptor mosaic. Science, New York, 221, 385-387.

Williams, D. R., MacLeod, D. 1. A. \& Hayhoe, M. M. (1981a). roveal tritanopia. Vision Research. 21. j341-1356.

Williams, D. R., MacLeod, D. 1. A. \& Hayhoe, M. M. (1981b). Punctate sensitivity of the blue-sensitive mechanism. Vision Research, 21, 1357-1375.

Willmer, E. N. \& Wright, W. D. (1945). Colour sensitivity of the fovea centralis. Nature, London, 156, 119-121. 